International Mathematical Forum, Vol. 8, 2013, no. 20, 983 - 988 HIKARI Ltd, www.m-hikari.com

\title{
Some Identities on the Generalized $q$-Euler Polynomials with Weak Weight
}

\author{
C. S. Ryoo \\ Department of Mathematics \\ Hannam University, Daejeon 306-791, Korea
}

Copyright (c) 2013 C. S. Ryoo. This is an open access article distributed under the Creative Commons Attribution License, which permits unrestricted use, distribution, and reproduction in any medium, provided the original work is properly cited.

\begin{abstract}
In this paper, we study the symmetry for generalized $q$-Euler numbers $E_{n, \chi, q}^{(\alpha)}$ and polynomials $E_{n, \chi, q}^{(\alpha)}(x)$ with weak weight $\alpha$. We obtain some interesting identities of the power sums and generalized $q$-Euler polynomials $E_{n, \chi, q}^{(\alpha)}(x)$ using the symmetric properties for the $p$-adic invariant $q$-integral on $\mathbb{Z}_{p}$.
\end{abstract}

Mathematics Subject Classification: 11B68, 11S40, 11S80

Keywords: Euler numbers and polynomials, generalized $q$-Euler numbers and polynomials, symmetric properties, power sums

\section{Introduction}

Throughout this paper we use the following notations. By $\mathbb{Z}_{p}$ we denote the ring of $p$-adic rational integers, $\mathbb{Q}$ denotes the field of rational numbers, $\mathbb{Q}_{p}$ denotes the field of $p$-adic rational numbers, $\mathbb{C}$ denotes the complex number field, and $\mathbb{C}_{p}$ denotes the completion of algebraic closure of $\mathbb{Q}_{p}$. Let $\nu_{p}$ be the normalized exponential valuation of $\mathbb{C}_{p}$ with $|p|_{p}=p^{-\nu_{p}(p)}=p^{-1}$. When one talks of $q$-extension, $q$ is considered in many ways such as an indeterminate, a complex number $q \in \mathbb{C}$, or $p$-adic number $q \in \mathbb{C}_{p}$. If $q \in \mathbb{C}$ one normally assume that $|q|<1$. If $q \in \mathbb{C}_{p}$, we normally assume that $|q-1|_{p}<p^{-\frac{1}{p-1}}$ so that $q^{x}=\exp (x \log q)$ for $|x|_{p} \leq 1$ (see [1-9]). Throughout this paper we use the notation:

$$
[x]_{q}=\frac{1-q^{x}}{1-q}, \quad[x]_{-q}=\frac{1-(-q)^{x}}{1+q} .
$$


Hence, $\lim _{q \rightarrow 1}[x]=x$ for any $x$ with $|x|_{p} \leq 1$ in the present $p$-adic case.

Let $U D\left(\mathbb{Z}_{p}\right)$ be the space of uniformly differentiable function on $\mathbb{Z}_{p}$. For $g \in U D\left(\mathbb{Z}_{p}\right)$ the fermionic $p$-adic invariant $q$-integral on $\mathbb{Z}_{p}$ is defined by Kim as follows:

$$
I_{-q}(g)=\int_{\mathbb{Z}_{p}} g(x) d \mu_{-q}(x)=\lim _{N \rightarrow \infty} \frac{1}{\left[p^{N}\right]_{-q}} \sum_{x=0}^{p^{N}-1} g(x)(-q)^{x}, \text { see }[1,2] .
$$

If we take $g_{n}(x)=g(x+n)$ in (1.1), then we see that

$$
q^{n} I_{q}\left(g_{n}\right)+(-1)^{n-1} I_{q}(g)=[2]_{q} \sum_{l=0}^{n-1}(-1)^{n-1-l} q^{l} g(l) .
$$

Note that

$$
\lim _{q \rightarrow 1} I_{-q}(g)=I_{-1}(g)=\int_{\mathbb{Z}_{p}} g(x) d \mu_{-1}(x) .
$$

Let a fixed positive integer $d$ with $(p, d)=1$, set

$$
\begin{aligned}
& X=X_{d}=\lim _{N}\left(\mathbb{Z} / d p^{N} \mathbb{Z}\right), \quad X_{1}=\mathbb{Z}_{p}, \quad X^{*}=\bigcup_{\substack{0<a<d p \\
(a, p)=1}} a+d p \mathbb{Z}_{p}, \\
& a+d p^{N} \mathbb{Z}_{p}=\left\{x \in X \mid x \equiv a \quad\left(\bmod d p^{N}\right)\right\},
\end{aligned}
$$

where $a \in \mathbb{Z}$ satisfies the condition $0 \leq a<d p^{N}$ (cf. [1, 2, 3, 4, 5, 6]). In [8], we introduced generalized $q$-Euler numbers $E_{n, \chi, q}^{(\alpha)}$ and polynomials $E_{n, \chi, q}^{(\alpha)}(x)$ attached to $\chi$. Let $\chi$ be the primitive Dirichlet character with conductor $d \in \mathbb{N}$ with $d \equiv 1(\bmod 2)$. We assume that $\alpha \in \mathbb{Z}$ and $q \in \mathbb{C}_{p}$ with $|q-1|_{p}<1$. Let $g(y)=\chi(y) e^{(y+x) t}$. By (1.1), we derive

$$
\begin{aligned}
\int_{X} \chi(y) e^{(y+x) t} d \mu_{-q^{\alpha}}(y) & =\frac{[2]_{q^{\alpha}} \sum_{a=0}^{d-1} \chi(a)(-1)^{a} q^{\alpha a} e^{a t}}{q^{\alpha d} e^{d t}+1} e^{x t} \\
& =\sum_{n=0}^{\infty} E_{n, \chi, q}^{(\alpha)}(x) \frac{t^{n}}{n !}
\end{aligned}
$$

By using Taylor series of $e^{(y+x) t}$ in the above equation (1.3), we obtain

$$
\sum_{n=0}^{\infty}\left(\int_{X} \chi(y)(y+x)^{n} d \mu_{-q^{\alpha}}(y)\right) \frac{t^{n}}{n !}=\sum_{n=0}^{\infty} E_{n, \chi, q}^{(\alpha)}(x) \frac{t^{n}}{n !} .
$$

By comparing coefficients of $\frac{t^{n}}{n !}$ in the above equation, we have the Witt formula for the generalized $q$ - Euler polynomials attached to $\chi$ as follows: 
Theorem 1.1 For positive integers $n$, we have

$$
E_{n, \chi, q}^{(\alpha)}(x)=\int_{X} \chi(y)(y+x)^{n} d \mu_{-q^{\alpha}}(y)
$$

Observe that for $x=0$, the equation (1.4) reduces to (1.5).

Corollary 1.2 For positive integers n, we have

$$
E_{n, \chi, q}^{(\alpha)}=\int_{X} \chi(y) y^{n} d \mu_{-q^{\alpha}}(y)
$$

By (1.4) and (1.5), we have the following theorem.

Theorem 1.3 For positive integers n, we have

$$
E_{n, \chi, q}^{(\alpha)}(x)=\sum_{l=0}^{n}\left(\begin{array}{l}
n \\
l
\end{array}\right) E_{l, \chi, q}^{(\alpha)} x^{n-l} .
$$

\section{Some identities for generalized $q$-Euler poly- nomials}

In this section, we assume that $q \in \mathbb{C}_{p}$. We obtain some interesting identities of the power sums and generalized $q$-Euler polynomials $E_{n, \chi, q}^{(\alpha)}(x)$ using the symmetric properties for the $p$-adic invariant $q$-integral on $\mathbb{Z}_{p}$. If $n$ is odd from (1.2), we obtain

$$
q^{n} I_{q}\left(g_{n}\right)+I_{q}(g)=[2]_{q} \sum_{l=0}^{n-1}(-1)^{l} q^{l} g(l) .
$$

Substituting $g(x)=\chi(x) e^{x t}$ into the above, we obtain

$$
\begin{aligned}
& q^{\alpha n d} \int_{X} \chi(x+n d) e^{(x+n d) t} d \mu_{-q^{\alpha}}(x)+\int_{X} \chi(x) e^{x t} d \mu_{-q^{\alpha}}(x) \\
& =[2]_{q^{\alpha}} \sum_{j=0}^{n d-1}(-1)^{j} \chi(j) q^{\alpha j} e^{j t} .
\end{aligned}
$$

For $k \in \mathbb{Z}_{+}$, let us define the $p$-adic functional $T_{k, \chi, q}^{(\alpha)}(n)$ as follows:

$$
T_{k, \chi, q}^{(\alpha)}(n)=\sum_{l=0}^{n}(-1)^{l} \chi(l) q^{\alpha l} l^{k} .
$$


After some elementary calculations, we have

$$
\begin{aligned}
& q^{\alpha n d} \int_{X} \chi(x) e^{(x+n d) t} d \mu_{-q^{\alpha}}(x)+\int_{X} \chi(x) e^{x t} d \mu_{-q^{\alpha}}(x) \\
& =\left(1+q^{\alpha n d} e^{n d t}\right) \frac{[2]_{q^{\alpha}} \sum_{a=0}^{d-1} \chi(a)(-1)^{a} q^{\alpha a} e^{a t}}{q^{\alpha d} e^{d t}+1} .
\end{aligned}
$$

From the above, we get

$$
\begin{aligned}
q^{\alpha n d} & \int_{X} \chi(x) e^{(x+n d) t} d \mu_{-q^{\alpha}}(x)+\int_{X} \chi(x) e^{x t} d \mu_{-q^{\alpha}}(x) \\
& =\frac{2 \int_{X} \chi(x) e^{x t} d \mu_{-q^{\alpha}}(x)}{\int_{\mathbb{Z}_{p}} q^{\alpha n d x} e^{n d t x} d \mu_{-1}(x)} .
\end{aligned}
$$

By (2.2), (2.3), and (2.4), we arrive at the following theorem:

Theorem 2.1 Let $n$ be odd positive integer. Then we obtain

$$
\frac{\int_{X} \chi(x) e^{x t} d \mu_{-q^{\alpha}}(x)}{\int_{\mathbb{Z}_{p}} q^{\alpha n d x} e^{n d t x} d \mu_{-1}(x)}=\sum_{m=0}^{\infty}\left(\frac{[2]_{q^{\alpha}}}{2} T_{m, \chi, q}^{(\alpha)}(n d-1)\right) \frac{t^{m}}{m !} .
$$

Let $w_{1}$ and $w_{2}$ be odd positive integers. By Theorem 2.1, and after some elementary calculations, we have the following theorem.

Theorem 2.2 Let $w_{1}$ and $w_{2}$ be odd positive integers. Then we have

$$
\frac{\int_{X} \chi(x) e^{w_{2} x t} d \mu_{-q^{w_{2}}}(x)}{\int_{\mathbb{Z}_{p}} q^{w_{1} w_{2} d x} e^{w_{1} w_{2} t d x} d \mu_{-1}(x)}=\frac{[2]_{q^{w_{2}}}}{2} \sum_{m=0}^{\infty}\left(T_{m, \chi, q}^{\left(w_{2}\right)}\left(w_{1} d-1\right) w_{2}^{m}\right) \frac{t^{m}}{m !} .
$$

Then we set

$$
S\left(w_{1}, w_{2}\right)=\frac{\int_{X} \int_{X} \chi\left(x_{1}\right) \chi\left(x_{2}\right) e^{\left(w_{1} x_{1}+w_{2} x_{2}+w_{1} w_{2} x\right) t} d \mu_{-q^{w_{1}}}\left(x_{1}\right) d \mu_{-q^{w_{2}}}\left(x_{2}\right)}{\int_{\mathbb{Z}_{p}} q^{w_{1} w_{2} d x} e^{w_{1} w_{2} d x t} d \mu_{-1}(x)} .
$$

By $S\left(w_{1}, w_{2}\right)$ and Theorem 2.2, after elementary calculations, we obtain

$$
\begin{aligned}
& S\left(w_{1}, w_{2}\right) \\
& =\left(\int_{X} \chi\left(x_{1}\right) e^{\left(w_{1} x_{1}+w_{1} w_{2} x\right) t} d \mu_{-q^{w_{1}}}\left(x_{1}\right)\right)\left(\frac{\int_{X} \chi\left(x_{2}\right) e^{x_{2} w_{2} t} d \mu_{-q^{w_{2}}}\left(x_{2}\right)}{\int_{\mathbb{Z}_{p}} q^{w_{1} w_{2} d x} e^{w_{1} w_{2} d x t} d \mu_{-1}(x)}\right) \\
& =\left(\sum_{m=0}^{\infty} E_{m, \chi, q}^{\left(w_{1}\right)}\left(w_{2} x\right) w_{1}^{m} \frac{t^{m}}{m !}\right)\left(\frac{[2]_{q^{w_{2}}}}{2} \sum_{m=0}^{\infty} T_{m, \chi, q}^{\left(w_{2}\right)}\left(w_{1} d-1\right) w_{2}^{m} \frac{t^{m}}{m !}\right) .
\end{aligned}
$$

By using Cauchy product in the above, we obtain

$$
S\left(w_{1}, w_{2}\right)=\sum_{m=0}^{\infty}\left(\frac{[2]_{q^{w_{2}}}}{2} \sum_{j=0}^{m}\left(\begin{array}{c}
m \\
j
\end{array}\right) E_{j, \chi, q}^{\left(w_{1}\right)}\left(w_{2} x\right) w_{1}^{j} T_{m-j, \chi, q}^{\left(w_{2}\right)}\left(w_{1} d-1\right) w_{2}^{m-j}\right) \frac{t^{m}}{m !}
$$


From the symmetry of $S\left(w_{1}, w_{2}\right)$ in $w_{1}$ and $w_{2}$, we also see that

$$
\begin{aligned}
& S\left(w_{1}, w_{2}\right) \\
& =\left(\int_{X} \chi\left(x_{2}\right) e^{\left(w_{2} x_{2}+w_{1} w_{2} x\right) t} d \mu_{-q^{w_{2}}}\left(x_{2}\right)\right)\left(\frac{\int_{X} \chi\left(x_{1}\right) e^{x_{1} w_{1} t} d \mu_{-q^{w_{1}}}\left(x_{1}\right)}{\int_{\mathbb{Z}_{p}} q^{w_{1} w_{2} d x} e^{w_{1} w_{2} d x t} d \mu_{-1}(x)}\right) \\
& =\left(\sum_{m=0}^{\infty} E_{m, \chi, q}^{\left(w_{2}\right)}\left(w_{1} x\right) w_{2}^{m} \frac{t^{m}}{m !}\right)\left(\frac{[2]_{q^{w_{1}}}}{2} \sum_{m=0}^{\infty} T_{m, \chi, q}^{\left(w_{1}\right)}\left(w_{2} d-1\right) w_{1}^{m} \frac{t^{m}}{m !}\right) .
\end{aligned}
$$

Thus we obtain

$$
S\left(w_{1}, w_{2}\right)=\sum_{m=0}^{\infty}\left(\frac{[2]_{q^{w_{1}}}}{2} \sum_{j=0}^{m}\left(\begin{array}{c}
m \\
j
\end{array}\right) E_{j, \chi, q}^{\left(w_{2}\right)}\left(w_{1} x\right) w_{2}^{j} T_{m-j, \chi, q}^{\left(w_{1}\right)}\left(w_{2} d-1\right) w_{1}^{m-j}\right) \frac{t^{m}}{m !} .
$$

Thus we arrive at the following theorem:

Theorem 2.3 Let $w_{1}$ and $w_{2}$ be odd positive integers. Then we have

$$
\begin{aligned}
& {[2]_{q^{w_{1}}} \sum_{j=0}^{m}\left(\begin{array}{c}
m \\
j
\end{array}\right) w_{1}^{m-j} w_{2}^{j} E_{j, \chi, q}^{\left(w_{2}\right)}\left(w_{1} x\right) T_{m-j, \chi, q}^{\left(w_{1}\right)}\left(w_{2} d-1\right)} \\
& =[2]_{q^{w_{2}}} \sum_{j=0}^{m}\left(\begin{array}{c}
m \\
j
\end{array}\right) w_{1}^{j} w_{2}^{m-j} E_{j, \chi, q}^{\left(w_{1}\right)}\left(w_{2} x\right) T_{m-j, \chi, q}^{\left(w_{2}\right)}\left(w_{1} d-1\right),
\end{aligned}
$$

where $E_{k, \chi, q}^{\left(w_{1}\right)}(x)$ and $T_{m, \chi, q}^{\left(w_{1}\right)}(k)$ denote generalized $q$-Euler polynomials with weak weight $w_{1}$ and $p$-adic functional, respectively.

By Theorem 1.3 and Theorem 2.3, we have the following corollary.

Corollary 2.4 Let $w_{1}$ and $w_{2}$ be odd positive integers. Then we obtain

$$
\begin{aligned}
& {[2]_{q^{w_{1}}} \sum_{j=0}^{m} \sum_{k=0}^{j}\left(\begin{array}{c}
m \\
j
\end{array}\right)\left(\begin{array}{l}
j \\
k
\end{array}\right) w_{1}^{m-k} w_{2}^{j} E_{k, \chi, q}^{\left(w_{2}\right)} T_{m-j, \chi, q}^{\left(w_{1}\right)}\left(w_{2} d-1\right) x^{j-k}} \\
& =[2]_{q^{w_{2}}} \sum_{j=0}^{m} \sum_{k=0}^{j}\left(\begin{array}{c}
m \\
j
\end{array}\right)\left(\begin{array}{l}
j \\
k
\end{array}\right) w_{1}^{j} w_{2}^{m-k} E_{k, \chi, q}^{\left(w_{1}\right)} T_{m-j, \chi, q}^{\left(w_{2}\right)}\left(w_{1} d-1\right) x^{j-k} .
\end{aligned}
$$

If we take $x=0$ in Theorem 2.3, we also derive the interesting identity for generalized $q$-Euler numbers with weak weight as follows:

Corollary 2.5 Let $w_{1}$ and $w_{2}$ be odd positive integers. Then we obtain

$$
\begin{aligned}
& {[2]_{q^{w_{1}}} \sum_{j=0}^{m}\left(\begin{array}{c}
m \\
j
\end{array}\right) w_{1}^{m-j} w_{2}^{j} E_{j, \chi, q}^{\left(w_{2}\right)} T_{m-j, \chi, q}^{\left(w_{1}\right)}\left(w_{2} d-1\right)} \\
& =[2]_{q^{w_{2}}} \sum_{j=0}^{m}\left(\begin{array}{c}
m \\
j
\end{array}\right) w_{1}^{j} w_{2}^{m-j} E_{j, \chi, q}^{\left(w_{1}\right)} T_{m-j, \chi, q}^{\left(w_{2}\right)}\left(w_{1} d-1\right) .
\end{aligned}
$$


By substituting Taylor series of $e^{x t}$ into (2.2), we obtain

$$
\begin{aligned}
& \sum_{m=0}^{\infty}\left(q^{\alpha n d} \int_{X} \chi(x+n d)(x+n d)^{m} d \mu_{-q^{\alpha}}(x)+\int_{X} \chi(x) x^{m} d \mu_{-q^{\alpha}}(x)\right) \frac{t^{m}}{m !} \\
& =\sum_{m=0}^{\infty}\left([2]_{q^{\alpha}} \sum_{j=0}^{n d-1}(-1)^{j} \chi(j) q^{\alpha j} j^{m}\right) \frac{t^{m}}{m !} .
\end{aligned}
$$

By comparing coefficients $\frac{t^{m}}{m !}$ in the above equation and $(2.3)$, we have the following theorem.

Theorem 2.6 Let $\chi$ be the primitive Dirichlet character with conductor $d \in \mathbb{N}$ with $d \equiv 1(\bmod 2)$. Then we have

$$
T_{m, \chi, q}^{(\alpha)}(n d-1)=\frac{q^{\alpha n d} E_{m, \chi, q}^{(\alpha)}(n d)+E_{m, \chi, q}^{(\alpha)}}{[2]_{q^{\alpha}}} .
$$

\section{References}

[1] T. Kim, q-Volkenborn integration, Russ. J. Math. Phys., 9(2002), 288299.

[2] T. Kim, $q$-Euler numbers and polynomials associated with $p$-adic $q$ integrals, J. Nonlinear Math. Phys., 14(2007), 15-27.

[3] T. Kim, J. Choi, Y-.H. Kim, C. S. Ryoo, A Note on the weighted p-adic $q$-Euler measure on $\mathbb{Z}_{p}$, Advan. Stud. Contemp. Math., 21(2011), 35-40.

[4] H. Y. Lee, N. S. Jung, and C. S. Ryoo, A Note on the $q$-Euler Numbers and Polynomials with Weak Weight $\alpha$, Journal of Applied Mathematics, 2011(2011), Art ID 497409, 14pp.

[5] C. S. Ryoo, A note on the weighted $q$-Euler numbers and polynomials, Advan. Stud. Contemp. Math., 21(2011), 47-54.

[6] C. S. Ryoo, A Note on the Twisted $q$-Euler Numbers and Polynomials with Weak Weight $\alpha$, Adv. Studies Theor. Phys., 6(2012), 1109 - 1116.

[7] C. S. Ryoo, An Identity of the $(h, q)$-Euler Polynomials Associated with the $p$-Adic $q$-Integrals on $\mathbb{Z}_{p}$, Int. Journal of Math. Analysis, 7(2013), $315-321$.

[8] C. S. Ryoo, On the generalized twisted $q$-Euler numbers and polynomials with weak weight $\alpha$, Adv. Studies Theor. Phys., 7(2013), 245 - 251

Received: April 3, 2013 